\title{
The Combustion Results of Suspension Water Coal Fuel in the "Teplotron" Type Boiler
}

\author{
Vasiliy I. Murko a, Vladimir I. Fedyaeva, \\ Yury E. Proshunin ${ }^{a}$, Alexander A. Pochechueva, \\ Viktor I. Karpenok ${ }^{\mathrm{a}}$ and Veniamin A. Hyamyalyainen ${ }^{\mathrm{b}}$ \\ "JSC "Scientific and Production Enterprise "Sibecotechnika" \\ 2 Kommunarov road, Novokuznetsk, 654000, Russia \\ ${ }^{b}$ Kuzbass State Technical University named after T.F. Gorbachev \\ 28 Vesennyaya Str., Kemerovo, 650000, Russia
}

Received 29.11.2017, received in revised form 07.03.2018, accepted 02.05.2018

A serious problem for coal production is the utilization of coal mining waste and coal enrichment. In different countries, the best specialists in this field are trying to find the solution of this problem. In the United States, France and China, the technology of preparation and burning of water-coal suspensions (WCF) for the utilization of fine-dispersed wastes of coal enrichment and coal slurries has been successfully used. The technology of WCF burning was developed in Russia. It was our country where the technology was first invented that allows to burn water-coal suspensions without preliminary dehydration and, most importantly, using coal for preparation of a coal-water mixture rather than waste of its extraction and enrichment.

Keywords: suspension water coal fuel, boiler "Teplotron", vortex combustion system.

Citation: Murko V.I., Fedyaev V.I., Proshunin Yu.E., Pochechuev A.A., Chernykh D.A., Hyamyalyainen V.A. The combustion results of suspension water coal fuel in the "Teplotron" type boiler, J. Sib. Fed. Univ. Eng. technol., 2018, 11(5), 560-566. DOI: $10.17516 / 1999-494 X-0054$.

(c) Siberian Federal University. All rights reserved

* Corresponding author E-mail address: sib_eco@kuz.ru 


\title{
Результаты сжигания
}

\section{суспензионного водоугольного топлива}

в котле типа «Теплотрон»

\author{
В.И. Мурко, В.И. Федяев ${ }^{\text {a }}$ Ю.Е. Прошунин, \\ А.А. Почечуев ${ }^{\text {a }}$ В.И. Карпенок ${ }^{\text {, }}$ В.А. Хямяляйнен ${ }^{6}$

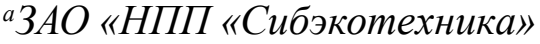 \\ Россия, 654000, Новокузнеик, проезд Коммунаров, 2 \\ ${ }^{6}$ Кузбасский государственный университет им. Т.Ф. Горбачева \\ Россия, 650000, Кемерово, ул. Весенняя, 28
}

Серьезной проблемой для угольного производства является утилизация отходов угледобычи и углеобогащцения. В разных странах решением этой проблемы занимаются лучшие специалисты в данной области. В США, Франциии и Китае успешно используют технологию приготовления и сжигания водоугольных суспензий (ВУТ) для утилизации тонкодисперсных отходов углеобогащения и угольных шламов. Получила развитие технология сжигания ВУТ и в России - именно в нашей стране впервые была изобретена технология, позволяющая сжигать водоугольные суспензии без предварительного обезвоживания и, главное, использующая для приготовления водоугольной смеси не уголь, а отходы его добычи и обогащения.

Ключевые слова: суспензионное водоугольное топливо, котел «Теплотрон», вихревая система сжигания.

\section{Введение}

В настоящее время около каждой крупной шахты, разреза или угольно-обогатительной фабрики образуются «хвосты» - громадные хранилища влажных или жидких отходов. Такие угольные отходы называются угольным шламом. Углеобогатительная фабрика или шахта средних размеров ежедневно выдает 300-600 т шлама - тех самых «хвостов», которые хранятся в многочисленных шламоотстойниках или породных отвалах. Однако в России и ряде других стран известна технология использования шламов в качестве суспензионного водоугольного топлива (ВУТ) [1-3].

Отношение к проблеме и соответствующие ассигнования на разработку технологий утилизации угольных шламов в разных странах определяются состоянием и степенью обеспечения местными энергоносителями, а также соотношением затрат на производимую на их основе энергию из добываемого или привозного угля, нефти и газа. Во многих странах целью программ по утилизации угольных шламов было обеспечение как экологической безопасности, так и экономической независимости [4].

\section{Результаты и обсуждения}

В рамках выполнения КузГТУ совместного российско-китайского проекта «Разработка эффективной технологии снижения содержания оксидов серы и азота, а также ртути в дымовых газах тепловых электростанций угольной генерации» по заказу Минобрнауки РФ был разработан и введен в эксплуатацию экспериментальный стенд для проведения исследований процессов приготовления и сжигания угольного топлива, в т.ч. суспензионного водоугольного 
топлива. Была создана установка сжигания различных топлив с измерением расхода и давления топлива, распыляющего агента и дутьевого воздуха, температуры горения, а также состава дымовых газов.

Согласно разработанной технологической схеме (рис. 1) на сжигание подается либо дробленый уголь (кл. 0-6(13) мм), либо суспензионное водоугольное топливо (ВУТ) [5-7]. Дробленый уголь подается на колосниковую решетку котла вручную. ВУТ дозировано подается в котел насосом объемного типа с регулируемым приводом. Для распыления водоугольного топлива в топке котла используется компрессорный воздух. Отходящие газы, проходя двухстадийную газоочистку, направляются в дымовую трубу $[8,9]$.

Для сжигания угля и ВУТ был разработан специализированный котел на базе известных котлов «Теплотрон», изготавливаемых на Новокузнецком котельном заводе [10].

Котел (рис. 2) состоит из вертикального цилиндрического корпуса 1 с водоохлаждаемой рубашкой 2 , дверцей 3 и крышкой 4 , колосниковой решетки 5 и зольника 6 . Наружная поверхность цилиндрического корпуса теплоизолирована. На боковой поверхности корпуса смонтированы горелочные устройства 7 с форсунками (на рисунке не показаны) и щелевые сопла 8 для тангенциальной подачи дутьевого воздуха. Внутренняя цилиндрическая поверхность камеры сгорания над колосниковой решеткой теплоизолирована термостойким материалом 9. Для подвода и отвода жидкого теплоносителя смонтированы соответственно патрубки 10 и 11. Для теплосъема оборудована система теплообмена 12 между горячими продуктами горения и теплоносителем. Корпус котла и система теплообмена связаны газоходом 13. Корпус котла с зольником и система теплообмена установлены на раме 14.

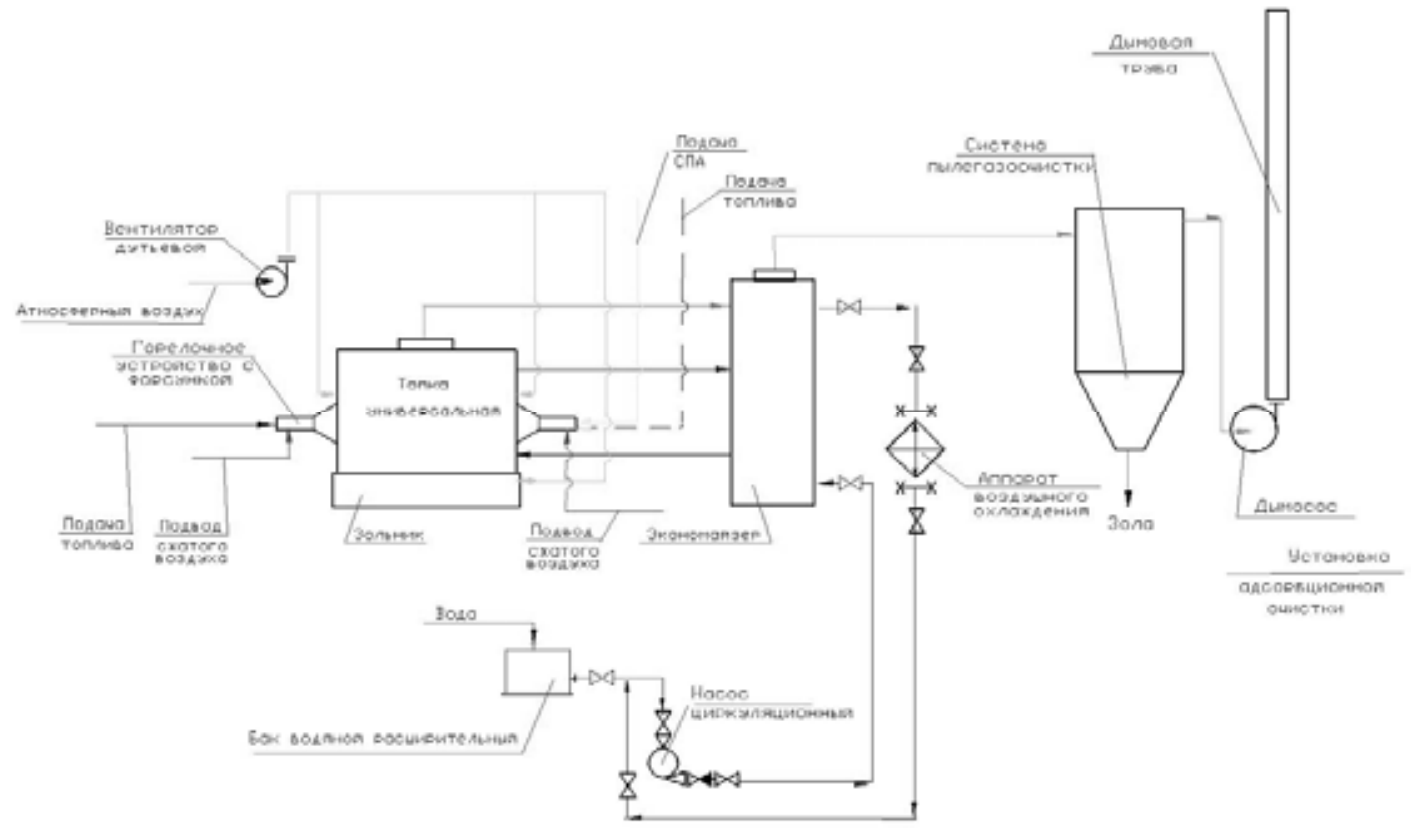

Рис. 1. Технологическая схема участка сжигания суспензионного угольного топлива (ВУТ)

Fig. 1. Technological scheme of the combustion of suspended coal fuel (WCF) 

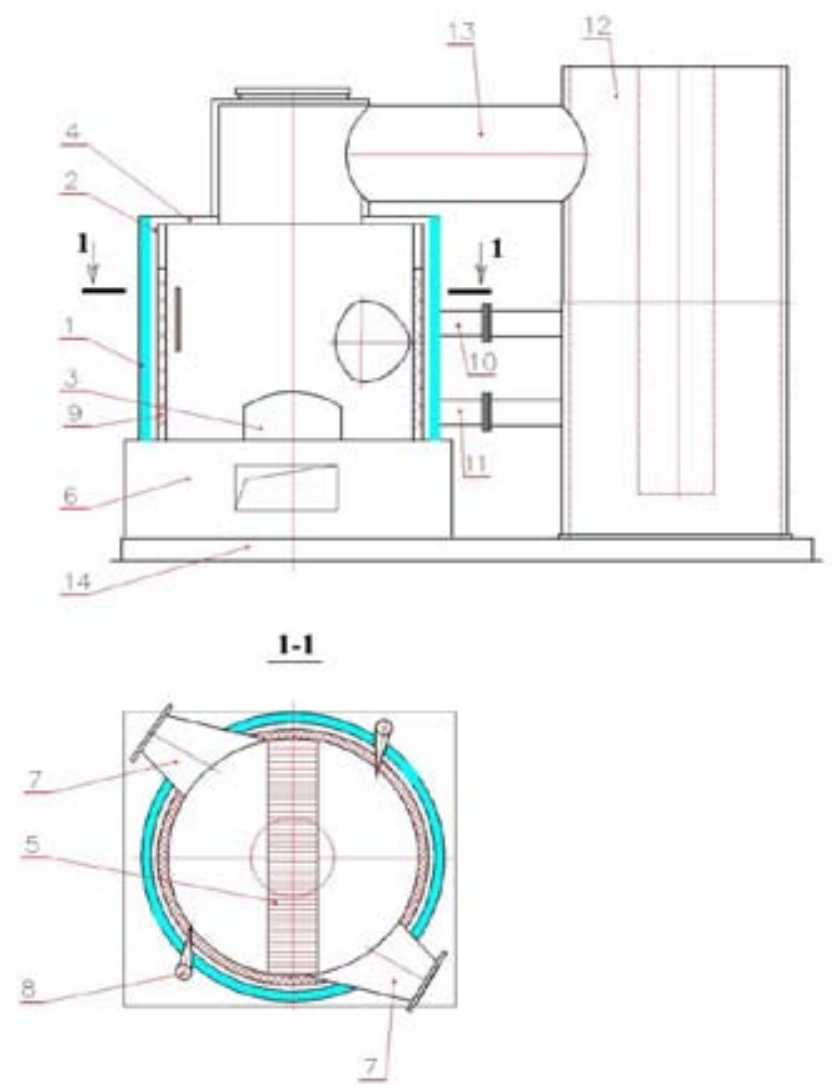

Рис. 2. Конструкция котла

Fig. 2. Design of the boiler

Котел работает следующим образом.

Первоначально производится прогрев внутренней полости корпуса котла - камеры сгорания, размещенной над колосниковой решеткой, за счет сжигания твердого топлива (уголь, дрова и др.) на колосниковой решетке. Затем начинается подача суспензионного водоугольного топлива [11, 12].

Суспензионное водоугольное топливо подается в котел насосом из емкости хранения (рис. 1) через форсунки горелочных устройств 7. В форсунки также подается распыляющий компрессорный воздух. Струи распыленного топлива подаются тангенциально условной поверхности внутри камеры сгорания корпуса 1 котла. Одновременно тангенциально в камеру сгорания подается воздух через щелевые сопла 8, ориентированные в том же направлении, что и горелочные устройства. За счет организации вихревого движения в корпусе котла осуществляется эффективное сгорание распыленного топлива [13]. Возможные несгоревшие крупные частицы угля выпадают на колосниковую решетку 5 , где догорают, создавая при этом дополнительную «подсветку» в зоне горения распыленного топлива. Крупные частицы золы и шлаковые отложения, накапливаемые на колосниковой решетке, периодически разгружаются в зольник 6. Образующиеся при сгорании топлива горячие газы через внутреннюю полость крышки 4 котла и газоход 13 поступают в систему теплообмена 12. В результате 
Таблица 1. Результаты испытаний

Table1 1. Test results

\begin{tabular}{|c|c|c|}
\hline Наименование показателей & По проекту & Фактически достигнутые \\
\hline Теплопроизводительность котла, МВт & 0,63 & 0,65 \\
\hline Низшая теплота сгорания топлива, ккал/кг & $3000-3100$ & $2900 \div 3400$ \\
\hline Расход топлива, л/ч & 300 & $160 \div 370$ \\
\hline Температура в топке, ${ }^{\circ} \mathrm{C}$ & $1050 \pm 50$ & $1100 \pm 100$ \\
\hline Температура отходящих газов, ${ }^{\circ} \mathrm{C}$ & 180 & 180 \\
\hline Температура воды, ${ }^{\circ} \mathrm{C}$ : & & \\
\hline - на входе & 60 & 55 \\
\hline - на выходе & 80 & 87 \\
\hline К.П.Д. котла, \% & 0,85 & 0,86 \\
\hline
\end{tabular}

происходит теплообмен между горячими газами и жидким теплоносителем. Частичный теплообмен между продуктами горения и жидким теплоносителем происходит также в корпусе котла за счет наличия водоохлаждаемой рубашки 2. Надежная и эффективная работа котла обеспечивается также наличием адиабатических или близких к ним условий в зоне горения топлива [14].

Транспортирование дымовых газов из топки в экономайзер предусмотрено с помощью дымососа. После экономайзера дымовые газы поступают в двухступенчатый пылеуловитель, а затем через дымовую трубу выбрасываются в атмосферу [15].

В табл. 1 представлены результаты испытаний нового котла типа «Теплотрон», работающего как на угле, так и на ВУТ.

В процессе испытаний пилотной установки измерение расхода топлива осуществлялось электромагнитным расходомером «Взлет-ЭРСВ». Производительность подачи топлива в топку регулировалась таким образом, чтобы при полном сгорании топлива, имеющего определенную низшую теплоту сгорания (2681-3400 ккал/кг), в котле вырабатывалась тепловая энергия в количестве не более 0,65 МВт. Во время испытания экспериментальной установки расход топлива с учетом изменения низшей теплоты сгорания ВУТ изменялся в интервале от 205 до 370 кг/ч. Температура в топке составляла $1000-1100{ }^{\circ} \mathrm{C}$.

\section{Заключение}

Результаты работы созданного котла полностью подтвердили его проектные показатели. При этом были достигнуты высокие показатели по уровню мех- и химнедожога топлива (соответственно не более $5 \%$ и 80 мг/м³ , что существенно меньше предельно допустимых значений).

Работа выполнена при финансовой поддержке Минобрнауки России в рамках реализации Федеральной целевой программы «Исследования и разработки по приоритетным направлениям развития научно-технологического комплекса России на 2014-2020 годы", по Соглашению № 14.583.21.0004 о предоставлении субсидии от 16 июля 2014 г. Уникальный 
идентификатор научных исследований (проекта) RFMEFI58314X0004, а также при финансовой поддержке РФФИ и Правительства Красноярского края в рамках научных проектов №̄№ 17-48-240386, 18-48-242001 и 18-41-242004.»

\section{Список литературы}

[1] Wan E.I., Fraser M.D., Logan C.N. Low sulphur coal-water fuel to retrofit a coal-fired to comply with US clean air act ammendments of 1990. In: Proceedings og yhe IEA-CLM. Clearwater, FL, USA, (26 April 1993), Paris, France, International Energy, 1993, 22.

[2] Ashworth R.A., Melick T.A., Morrison D.K., Battista J.J. Electric utility CWS firing options to reduce $\mathrm{NO}_{\mathrm{x}}$ emissions, Twenty Third International Technical Conference on Coal Utilization \& Fuel Systems, Coal \& Slurry Technology Association and ASME-FACT, Clearwater, Florida, 1998, 719-730.

[3] Morrison J.D., Scaroni A.W., Battista J.J. The use of coal slurries for production of coalwater fuel. XIII International Coal Preparation Congress. Brisbane, Australia, 4-10 Oct. 1998. 643-645.

[4] Alaa M. Musalam and Abdel Fattah A. Qaraman. The thermal behavior of the coal-water fuel (CWF). International Journal of Energy and Environmental Research, 2016, 4(3), 27-36.

[5] Murko V.I., Karpenok V.I., Senchurova Yu.A., Khyamyalyainen V.A., Tailakov O.V. Study of sulfur oxide reduction during combustion of coal-water slurry. Coal in the $21^{\text {st }}$ Century: Mining, Processing and Safety, 2016, 297-300.

[6] Murko V.I., Fedyaev V.I., Karpenok V.I., Zasypkin I.M., Senchurova Y.A., Riesterer A. Investigation of the spraying mechanism and combustion of the suspended coal fuel. Thermal Science, 2015, 19(1), 243-251.

[7] Murko V.I., Puzyryov E.M., Karpenok V.I., Fedyaev V.I., Baranova M.P. The Usage Of Boilers With A Vortex Furnace For Burning Enrichment Products And Deballasting Coal. XVIII International Coal Preparation Congress 28 June-01 July 2016, Saint-Petersburg, Russia.

[8] Zasypkin I.M. Systems of ignition and combustion stabilization for water-coal fuel. Thermal science, 2012, 16(4), 1229-1238.

[9] Murko V.I., Fedyaev V.I., Aynetdinov H.L., Baranova M.P. Environmentally Clean Technology of Fine Waste Coal Utilization. XVII International coal preparation congress, Istanbul, Turkey, 1-6 Oct. 2013.

[10] Patent No. 2145038. M.cl. F 23 Q 5/00. Method of Combustion and Combustion Stabilization of the Water-Coal Fuel in the Settling Chamber (in Russian), V.I. Murko, M.P. Fomicheva, A.N. Timoshevskiy, I.M. Zasypkin, et al. No. 97120914/06. Appl. 03.12.97. Published on 27.01.2000, Bulletin No. 3.

[11] Prudhon M.G. Boiler plant with a circulating liquid phase of 320 MWth, France unification project collieries SODELIF. Helsinki, Finlande, 10-13 May 1993.

[12] Biletskyy V., Sergeyev P. and Krut O. Fundamentals of highly loaded coal-water slurries. Mining of Mineral Deposits. Taylor \& Francis Group, London, 2013, 105-113.

[13] Mochalov S.P., Rybenko I.A., Ermakova L.A. Mechanism and Mathematical Modeling of Coal-Water Slurry Combustion in Swirl Adiabatic Combustion Chamber. World Applied Sciences Journal, 2012, 19(1), 20-25.

$$
-565-
$$


[14] Kijo-Kleczkowska A. Analysis of cyclic combustion of the coal-water suspension Archives of Thermodynamics, 2011, 32(1). 45-75

[15] Kijo-Kleczkowska A. Combustion of coal-water suspensions, Fuel, 2011, 90(2), 865-77. 